\author{
BELÉN LEAL HURTADO* \\ RUTH BETTY ARAGÓN AGUILAR **
}

Fundación Universitaria del Área Andina (Bogotá, Colombia)

\title{
Relaciones externas al aula: experiencia en la Fundación Universitaria del Área Andina ${ }^{* * *}$
}

\author{
External relations on the classroom: Experiences area \\ Andina University Foundation
}
Relações externas à sala de aula: experiência na fundação universitária da área andina

\footnotetext{
* Socióloga. Magister en Educación. Especialista en Docencia y Proyectos Educativos Institucionales. Docente de Tiempo Completo del Departamento de Humanidades de la Fundación Universitaria del Área Andina. ORCID oooo-00o2-77193109. Correo Electrónico: bleal@areandina.edu.co

** Psicóloga. Magister en Educación con Énfasis en Docencia Universitaria. Especialista en Pedagogía para el Desarrollo del Aprendizaje Autónomo. Especialista en Psicología Médica y de la salud. Docente de Tiempo Completo del Departamento de Humanidades de la Fundación Universitaria del Área Andina. Correo Electrónico: raragon@areandina.edu.co

*** Artículo resultado del Proyecto de Investigación realizado entre febrero y diciembre de 2015, financiado por la Fundación Universitaria del Área Andina. Artículo de investigación recibido el 15-11-2016 y aprobado 21-06-2017.
} 


\section{Cómo citar}

Leal Hurtado, B. y Aragón Aguilar, R. B. (2017).

Relaciones externas al aula: experiencia en la Fundación

Universitaria del Área Andina. Revista CS, 23, pp. 135-156.

DOI: http://dx.doi.org/10.18046/recs.i23.2314 
Este artículo es el resultado del proyecto de investigación realizado en el contexto académico. Su objetivo fue analizar la forma en que los y las estudiantes de la Fundación Universitaria del Área Andina conciben las relaciones de amistad, relaciones de pareja y manejo de conflictos externos al aula. Se desarrolló bajo un enfoque psicosocial de tipo cualitativo, exploratorio, no comparativo, con aplicación del método de grupos focales mixtos, a una población de 48 estudiantes entre 18 y 25 años, matriculados en el tercer semestre de la Facultad de Ciencias de la Salud: programas de Enfermería, Optometría y Terapia Respiratoria, y de la Facultad de Diseño, Comunicación y Bellas Artes, con los programas de: Diseño Gráfico, Diseño de Modas, Culinaria y Gastronomía, sede Bogotá. Se dividió en tres fases investigativas: primero, aproximación conceptual al tema; segundo, análisis e interpretación de los resultados decantados a través de las voces de los y las jóvenes, determinando las categorías de amistad, pareja y manejo de conflictos; tercero, discusión, conclusiones y recomendaciones.

\section{PALABRAS CLAVE:}

Relaciones interpersonales | amistad | pareja | conflictos

This article is the result of a research project conducted in the academic context. Its aim was to analyze how the students of the Area Andina University Foundation conceive relations of friendship, relationships and management of conflicts outside the classroom. It was developed under a psychosocial approach of qualitative and exploratory, not comparative, type with application of the method of mixed focus groups with a population of 48 students, between 18 and 25 years, enrolled in the third semester of the Faculty of Health Sciences: Nursing, Respiratory Therapy Optometry programs and the School of Design, Communication and Fine Arts, with programs: Graphic Design, Fashion Design, Culinary and Gastronomy in Bogotá. It was divided in three investigation phases. The first phase is about, conceptual approach to the subject. The second phase is about, analysis and 
interpretation of results decanted through the voices of young people, determining the categories of friendship, family and conflict management. In the last phase, discussion, conclusions and recommendations.

\section{KEYWORDS:}

Interpersonal relationships | friendship | couple | conflict

Este artigo é o resultado do projeto de pesquisa realizado no contexto acadêmico. Seu objetivo foi analisar a forma em que os estudantes da Fundación Universitaria del Área Andina concebem as relações de amizade, relações de casal e a maneira como lidar com conflitos externos à sala de aula. Desenvolveu-se com um foco psicossocial de tipo qualitativo, exploratório, não comparativo, com aplicação do método de grupos focais mistos, a uma população de 48 estudantes, entre 18 e 25 anos, matriculados no terceiro semestre da Faculdade de Ciências da Saúde: programas de Enfermagem, Optometria e Terapia Respiratória e da Faculdade de Design, Comunicação e Belas Artes, com os programas de: Design Gráfico, Design de Modas, Culinária e Gastronomia, sede Bogotá. Dividiu-se em três fases de pesquisa, primeiro, uma aproximação conceitual do tema. Segundo, análise e interpretação dos resultados decantados através das vozes dos jovens, determinando as categorias de amizade, casal e a maneira de lidar com os conflitos. Terceiro, discussão, conclusões e recomendações.

\section{PALAVRAS CHAVES:}

Relações interpessoais | amizade | casal | conflitos 


\section{Introducción}

La historia de la humanidad en torno a la forma en que se relacionan las personas se ha caracterizado por la ingeniosidad de compartir afinidades, similitudes, diferencias o conflictos en los modos de vida. Para desarrollar dichas relaciones se han utilizado diversas estrategias de adaptación al medio, lo que, podría afirmarse, se ha constituido en una manera de subsistir y a la vez enriquecer la condición de la vida humana con plenitud intrapersonal e interpersonal.

Se entiende por relación interpersonal al conjunto de actitudes sociales por medio de las cuales los individuos construyen interacciones, empleando maneras propias, significativas o no, bien sea para sostenerlas o para finalizarlas; estas relaciones son mediadas por factores internos, tales como: la herencia biológica, la personalidad, la percepción, las creencias y los estados emocionales, y por factores externos como: el medio, el rol, el status y los procesos de socialización.

Las relaciones interpersonales son un aspecto insubordinado de la realidad. Los sentimientos personales, las avenencias emocionales, afectivas o de pareja de una relación son esenciales en la realización personal, forman parte de un proceso sistémico, significativo, necesario y relevante en el diario vivir, donde juega un papel fundamental el desarrollo integral de la persona. A través de ellas, los seres humanos se reafirman a sí mismos y reafirman a los otros en su ser, estar y convivir. Tanto la psicología como la sociología, con base en estudios de los estados emocionales y conductuales en el contexto de la realidad social, otorgan bases teóricas que sustentan las interacciones que establecen los seres humanos.

Con lo dicho, el escenario social hace propicio que en las relaciones se presenten conductas de acercamiento a través de diversos estilos de amistad, de pareja, con expresiones de unión, proximidad y solidez, donde los conflictos pueden valorarse y ser analizados por cada una de las partes conduciendo a situaciones estables y duraderas, y tendiendo a optimizarse cada vez más. Por otra parte, también pueden presentarse conductas de distanciamiento que implican ruptura, apatía e indiferencia, entorpeciendo la vida cotidiana e íntima hasta llegar a disolverse.

La vida académica es también un proceso de relación, ya que involucra la transferencia de conocimientos y valores. El tema de las relaciones interpersonales fuera del aula de clase, aunque no ha sido muy estudiado en la vida universitaria, se constituyó en el objetivo de investigación, con la finalidad de analizar la forma en que las y los estudiantes de la Fundación Universitaria del Área Andina conciben las relaciones de amistad, relaciones de pareja y manejo de conflictos externos al aula. 


\section{Categorías de investigación: aproximación conceptual}

A partir de la profundización del tema sobre las relaciones interpersonales fuera del aula de clase y el diálogo académico, se establecieron seis categorías de investigación; así: cultura de paz, relaciones de amistad, manejo de conflictos, emociones, percepción y relaciones de pareja. Posteriormente, se planeó y avaló un abanico de preguntas con cada una de las categorías descritas que, en prueba piloto, con grupos focales, se ponderaron, surgiendo las tres sobre las cuales gira el proyecto. A continuación, se puntualiza cada una de ellas.

\section{Relación de amistad}

Antropológicamente, las relaciones de amistad son dinámicas y se hallan condicionadas bajo aspectos culturales y sociales, e inciden de forma efectiva en la estructura social. Aparecen en todas las sociedades, son variables de acuerdo con las diferentes cosmovisiones, más o menos ritualizadas, y desempeñan tareas, oficios, obligaciones con el otro y con el entorno. «La amistad es tan antigua, tan poderosa, tan sugerente y bella como el mismo ser humano puede ser. Y, como él, compleja y ambivalente. Deseada por todos, temida por algunos, lo cierto es que nadie querría vivir sin amigos, como el viejo Aristóteles escribió» (Romero, 2015: 1).

Carl Rogers (1980: 60) destaca cuatro características de la amistad como una relación afectiva sana: 1) La autenticidad, es decir, tiene que expresar claramente el modo de sentir sin máscaras ni reservas. 2) La cordialidad, que consiste en una aceptación y consideración incondicionales, sin pretender que los demás hagan lo que uno quiere. 3) La empatía, que es la capacidad para comprender lo que la otra persona siente; a veces es preciso escuchar intensamente lo que dice, y otras se percibe sin necesidad de palabras. 4) La disposición de apertura hacia el otro, de compartir de puertas para afuera: las personas que más se abren suelen tener más amigos.

Muchas cualidades se atribuyen a la amistad, se constituyen en dimensiones esenciales de compartir con los otros y son un escenario consistente de relaciones mediadas por valores sociales que cultivan las actuaciones eficientes y eficaces de los individuos. Se desenvuelven libre y espontáneamente, sin determinación en el tiempo; se caracterizan por el aprecio, el apoyo, la comunicación, el afecto y comportamientos axiológicos sin que impliquen un compromiso de rol específico, tendiendo a manifestarse en todas las circunstancias que afecten la fraternidad. 


\section{Relación de pareja}

Se entiende como un estilo de vida cotidiano, significativo en las personas. La pareja se fundamenta en aspiraciones, deseos, proyectos, sueños y, en general, expectativas personales, psicobiológicas, sociales y culturales como parte del proyecto de vida.

Se advierte que la funcionalidad de la pareja se construye a partir de «dos personas procedentes de familias distintas, generalmente de distinto género, que deciden vincularse afectivamente para compartir un proyecto común, lo que incluye apoyarse y ofrecerse sucesos importantes mutuamente. Es un espacio propio que excluye a otros, pero que interactúa con el entorno social» (Campo y Linares, 2002: 45).

Un atributo de la relación de pareja hoy es la variabilidad de concepciones de género, creencias y roles que existen frente a ella; sin embargo, prima la mirada tradicional de considerarla como vínculo de amor, crecimiento, ideales y progreso. Además de conformar una identidad propia de pareja, conservando la personal, e idealmente compartiendo tareas y actividades en diversas situaciones.

\section{Manejo de conflictos}

Por lo general, se tiende a considerar el conflicto de una manera reduccionista negativa, invalidando el crecimiento y posibilidades que el mismo proporciona. «Los conflictos son consustanciales e ineludibles en la naturaleza humana. Por eso no pueden ni deben ser eliminados, podemos aprender a reconocerlos y hacer de ellos una oportunidad de transformación, aprendizaje y crecimiento» (Observatorio para la Paz, 2014:38).

El conflicto es el producto de la conducta relacional humana y del resultado de convivir en sociedad con diversas ópticas sobre la manera de percibir la realidad, entendiendo que el conflicto no necesariamente implica o justifica cualquier forma de violencia.

Desde la psicología, el conflicto puede entenderse como un proceso inherente al desarrollo del ser humano, en las dimensiones sociales, emocionales, ideológicas, espirituales, políticas, sexuales, éticas, religiosas y culturales. Se entiende también como un sentimiento doloroso, triste y emotivo, producido por la ansiedad entre deseos opuestos, diferencias y contradicciones. El conflicto es resultado de las interacciones sociales, el entramado de los grupos, los antagonismos de diverso orden. Caracterizado especialmente, por la querella de valores, los roles, el estatus, las relaciones políticas, de poder, de afiliación, todo lo cual, en la cúspide del mismo, altera la propia estructura de los grupos, a los miembros que la componen, e incluso afectando la organización de la sociedad. 
Con todo lo anterior, y teniendo identificadas las categorías en la investigación, se establece como objetivo: analizar la forma en que las y los estudiantes de la Fundación del Área Andina conciben las relaciones de amistad, relaciones de pareja y manejo de conflictos externos al aula.

\section{Metodología}

La presente investigación tiene un enfoque cualitativo y exploratorio. La población está conformada por estudiantes de la Facultad de Ciencias de la Salud, programas de Enfermería, Optometría y Terapia Respiratoria y la Facultad de Diseño, Comunicación y Bellas Artes, con los programas de Diseño Gráfico, Diseño de Modas, Culinaria y Gastronomía de la Fundación Universitaria del Área Andina, sede Bogotá, en el año 2015. La muestra es aleatoria simple, no probabilística, con la participación de 48 estudiantes, entre 18 y 25 años, matriculados en el tercer semestre. Para el estudio de campo, se seleccionó un promedio de 8 a 10 estudiantes por cada uno de los programas académicos descritos, aplicando la técnica de grupos focales mixtos, con entrevista semiestructurada a profundidad.

El proceso de las entrevistas a las categorías de análisis, se desarrolló con base en la técnica de destilación de información propuesta por el maestro Fernando Vásquez Rodríguez (2013), como se aprecia en el cuadro siguiente.

CUADRO 1

\begin{tabular}{l|l|}
\multicolumn{1}{c}{ Etapa } & \multicolumn{1}{c|}{ Actividad } \\
\hline Preparación & o. Criterios base para el análisis \\
\hline \multirow{3}{*}{ Clasificación y codificación } & 1. Transcripción de textos \\
\hline & 2. Clasificación de los textos \\
\hline Análisis de la información & 3. Análisis de pertinencia \\
\hline & 4. Selección o recorte \\
\hline Catgeorización y conceptualización & 5. Tamizaje de los recortes \\
\hline
\end{tabular}




\section{Resultados}

El proceso de análisis de los datos obtenidos a través del método Destilar la información, permitió, a partir de los textos originales de las entrevistas realizadas a los y las estudiantes, sujetos de la investigación, desglosar la información de cada una de las categorías de análisis: relaciones de amistad, relaciones de pareja y manejo de conflictos.

Para efectos de la fase de interpretación, a continuación, se incluyen las tablas clasificadas de los descriptores por analogía, en cada una de las tres categorías investigadas.

\section{Relaciones de Amistad}

TABLA 1 Listado de descriptores. Categoría Relaciones de Amistad

\section{Respuestas}

\section{DEFINICIÓN}

En apreciación de las y los estudiantes, el concepto de amigo se relacionó fuertemente con el establecimiento de vínculos: con otras y diversas personas. A quienes se quiere, respeta y comparte. Entre dos o más personas para apoyarse y comentar. Entre la familia. De pensamientos entre personas. De afecto con alguien con quien se tiene química. Lazos que se crean en la universidad.

Es compartir y hablar. Momentos claves que te aporta más de lo que hace. Todo lo que pasa. Es un tesoro muy valioso.

Relación interpersonal estrecha del mismo género. Entre dos personas. Se da en diferentes etapas de la vida. Se pueden contar cosas personales y laborales.

Es un complemento, un hermano, que lo une y fortalece.

Es algo efímero que depende de las circunstancias.

Con la persona que se habla, pero no se tiene compromiso.

1

1

\section{CARACTERÍSTICAS}

Como particularidades que caracterizan las relaciones de amistad, se encontraron las siguientes: que se puede confiar en las personas. Confianza y afinidad. Existe afinidad en la personalidad. Concordancia de pensamientos. Deben ser iguales, aunque sea un poco. Concordancia de pensamientos, por eso se genera la amistad. Se da después de muchos años. Debe haber respeto. Confianza y amistad. La amistad maneja la confianza. Se cataloga por la confianza y la afinidad. 
Se fundamenta en que las personas se complementen. Tengan visiones diferentes. Es un complemento.

3

Se puede encontrar en la universidad. Se va dando en las actividades externas a las académicas.

Se escoge la amistad. Se tiene que ver a quién se le da la confianza.

Debe dejarla entrar a la vida de uno. Uno aporta algo al otro para la vida.

2

En el colegio es con quien se comparte más tiempo y se conocen las familias.

Hay que conocer la familia de los amigos.

La amistad en la universidad es con quien solo se habla de trabajo.

Los amigos deben ser extrovertidos y alegres.

La amistad da consejos y deja que el otro decida.

La amistad en la academia es un pilar.

\section{QUÉ HACE UN AMIGO}

Es el que está con uno siempre. El que está con uno en las buenas y en las malas.

Está con uno así conozca los defectos.

Un amigo ayuda a superarse.

Un amigo se acompaña en locuras.

1

Un amigo respeta el pensar y tolera.

1

Un amigo no es solo intenciones, actúa.

El amigo soluciona los conflictos.

1

Los buenos amigos son los que no lo dejan morir a uno nunca.

1

\section{Subcategoría. Conceptualización}

Se deduce que las y los estudiantes definen ampliamente la amistad, dándole especial connotación al vínculo entre dos o más personas entre las que debe existir una química, situación que les permite mostrarse como son. Además, consideran que es una relación interpersonal afectiva y estrecha entre personas del mismo género, valiosa, de apoyo para contar cosas personales y laborales. Se da en diferentes etapas de la vida.

Se estima así que los amigos son parte imprescindible en la vida de las personas. La amistad es una prodigiosa expresión de vínculos, apertura al diálogo, condición de posibilidad de encuentro del «yo» con el «otro yo» donde se cultivan las relaciones afectuosas, virtudes y proyectos. 
Calleja (2011) retoma la teoría de Taylor y Altman, doctores en psicología social, quienes discurren sobre la manera en que las personas se comunican y difieren en la comprensión y entendimiento de las relaciones afectivas, pasando por un proceso inicial de frivolidad, quizá carente de metas, sueños e ideales, superándose con el tiempo a uno más consolidado que incluye la integralidad del ser.

En la amistad se presentan acercamientos en los que aparecen expresiones de solidez, unión, proximidad y química, donde las incomprensiones y dificultades pueden valorarse y analizarse por cada una de las partes, consolidando lazos afectivos más estrechos. Cabe resaltar, que, en escala inferior, los y las estudiantes valoran la amistad como un complemento de unión y fortaleza, como de hermanos; paralelamente, también la encuentran efímera y dependiente de las circunstancias, con la persona que se habla pero no se tiene compromiso.

\section{Subcategoría. Características}

Es relevante el atributo que le dan a la amistad basada en la confianza, la afinidad en relación con la personalidad, la concordancia de pensamientos y de visiones diferentes donde se complementan el uno al otro.

Es sentida la tendencia de los y las estudiantes a distinguir la amistad desde un punto de vista de los valores, donde las relaciones se justifican a través de propósitos humanos y hábitos sanos que ayudan a crecer y oxigenan la vida, como afirmara Cano (2008: 134) aludiendo a Francisco de Quevedo: «El amigo ha de ser como la sangre, que acude a la herida sin esperar que la llamen».

En menor escala, los y las estudiantes reconocen la amistad dentro de la universidad y fuera del aula de forma dual: de una parte, consideran que la academia es un pilar para establecer relaciones, de otra parte, es con quien solo se habla de trabajo en el estudio; sobresale también que los amigos deben ser extrovertidos, alegres, dan consejos y dejan que el otro decida.

\section{Subcategoría. Qué hace un amigo}

Reafirman de manera especial que un buen amigo es aquel que apoya, se preocupa y retroalimenta al otro en las buenas y en las malas; es confidente, lo ayuda a superarse. La amistad se da a pesar de que conozca los defectos. Aristóteles atribuye a la amistad: «la igualdad o semejanza entre los amigos, la necesidad de convivencia... alegrías y-sobre todo-penas, la permanencia, el esfuerzo, el crecimiento, la mutua corrección entre amigos, etc.» (Sellés, 2008: 147).

Por último, en esta categoría, en cuanto a los descriptores de menos asiduidad en la conversación con los y las estudiantes: estiman que un buen amigo es aquel que 
no lo deja morir a uno nunca, lo acompaña en locuras, ayuda a superar conflictos y más allá de las intenciones actúa, respeta y tolera.

\section{Relaciones de pareja}

TABLA 2 Listado de descriptores. Categoría Relaciones de Pareja

Respuestas

\section{DEFINICIÓN}

A criterio de las y los estudiantes, las relaciones de pareja se entienden como: unión de dos personas con las mismas características y gustos. Entrega que se da entre dos personas. Compartir su vida con esa persona.

Vínculo afectivo, en donde hay interés y entrega. Interés entre dos personas que sienten cariño.

Son amigos con ciertos beneficios. Es como tener una relación de amistad.

Es como un complemento que tiene uno con otra persona. Mucha afinidad.

Es su alma gemela.

Es un compromiso en donde se cumplen ciertas reglas.

Relación con otra persona que simpatice, tenga cualidades y llene sus expectativas.

\section{CARACTERÍSTICAS}

Consideran que la confianza y el cariño no son lo mismo en un amigo que en pareja. La relación de pareja es más grande. A lo largo del tiempo suple muchas necesidades que la amistad no cubre. La confianza puede ser igual que la que se tiene con un amigo, pero el trato con la pareja tiene unos beneficios.

La pareja así no tenga los mismos pensamientos debe complementarse y ayudar al otro a crecer. Le ayuda a aprender cosas nuevas. Soporta los gustos del otro.

Las mujeres son las que más pelean, el hombre cede así no sea culpable. Cede para no seguir peleando.

Trasciende la amistad. Va más allá de amistad.

Uno no debe someterse a las otras personas.

La relación de pareja conlleva amor.

El tener gustos diferentes lleva a una buena relación. 


\section{CONFLICTOS}

Para evitar complicaciones, una discusión de pareja, es mejor cambiar de tema.

Es bueno ceder en el momento del problema, para hablar cuando la situación pase.

En un conflicto de pareja sí es importante entender a la otra persona.

El conflicto se da en la pareja por el choque de actitudes.

Las discusiones en pareja tienen consecuencias más fuertes que pueden llevar a acabar con la relación.

\section{Subcategoría. Conceptualización}

Las relaciones de pareja consideradas como un aspecto vital de la esencia humana, son percibidas por los estudiantes como la entrega, unión e interés de dos personas con afinidad, similares características con las que se puedan compartir los mismos gustos. También es interesante para ellos obtener de dicha relación interpersonal ciertos beneficios, que dan como resultado bienestar; ayuda al crecimiento personal y sirve para compartir la vida.

La experiencia investigativa y estas aportaciones de las y los estudiantes, encuentran asidero en la teoría del intercambio social de Thibaut y Harold, para quienes el valor emocional de una relación está en mutua dependencia con las recompensas y las sanciones, a partir de las cuales las personas evalúan las interacciones, aceptando aquellas que aportan un beneficio, volviéndose positivas y duraderas en el tiempo; rechazando las que generan dolor o sufrimiento por considerarlas negativas y poco saludables.

Palacios (2004) citado por Morales (2005:3), afirma que: «Las personas buscan igualdad o equivalencia entre la razón de aportaciones y resultados en sus relaciones. A grosso modo, esta teoría propone que las personas luchan por la equidad en sus relaciones, y se sienten mal cuando perciben una injusticia...». Supuesto aplicable a las relaciones más íntimas como las de pareja.

Vale la pena aclarar que en escasa proporción los comentarios de los y las estudiantes, se orientan a entender la relación de pareja como el encuentro con el alma gemela, donde hay compromisos, se cumplen reglas, existe simpatía y la otra persona debe tener cualidades y llenar expectativas. 


\section{Subcategoría. Características}

Desde la perspectiva discursiva de los y las estudiantes, se parte de diferenciar entre el cariño que se le otorga a un amigo, respecto al que se brinda en la relación de pareja. Resaltan la confianza y la ayuda al crecimiento y aprendizaje de cosas nuevas, y que así no se tengan los mismos pensamientos o los mismos gustos, deben complementarse, al igual que suplir muchas necesidades que la amistad no cubre.

Sin embargo, como argumentan Garrido, Reyes, Torres y Ortega (2008), históricamente, la conformación de relaciones de pareja se realizaba con base en las dotes, el interés político de las familias y los recursos económicos, factores que aún siguen vigentes en algunas sociedades. En la sociedad contemporánea estas relaciones se rigen por libre determinación, por complementariedad o afinidad con base en gustos, metas, habilidades u objetivos que se comparten, pudiendo tanto el hombre como la mujer decidir a quién desean como compañero.

Ahora bien, se encontró una gama de opiniones menos recurrente en los y las estudiantes, donde expresan su sentir considerando que en las parejas existen gustos diferentes, pero llevan a una buena relación más íntima conduciendo al amor; además no debe haber relaciones de sometimiento.

\section{Subcategoría. Conflictos}

Es pertinente señalar aquí la ausencia de frecuencia en las voces de los y las estudiantes, debido a que todas las opiniones son disímiles, es decir, no hay reiteración en las visiones sobre el conflicto en las parejas.

Las apreciaciones identificadas en esta subcategoría fueron: cuando existe una discusión de pareja es bueno ceder en el momento del problema, cambiar de tema, entender a la pareja y evitar así complicaciones, ya que estas pueden tener graves consecuencias y generar rupturas. Existen tipos de relaciones caracterizadas por noviazgo, formar familia o de esposa, con la cual puede haber mayores logros y además mirar en ella lo que cada uno puede aportar. Por último, el conflicto se presenta en la pareja por la oposición de actitudes.

Se advierte en las respuestas que, por un lado, cuando las parejas tienen discusiones que les llevan a la búsqueda de una solución al conflicto, estas serán positivas y funcionales desde el punto de vista del desarrollo saludable de la pareja; por otro lado, puede ser que por falta de habilidades estratégicas la pareja no resuelva los conflictos, presentándose la disfuncionalidad hasta llegar a separarse. 


\section{Manejo de Conflictos}

TABLA 3 Listado de descriptores. Categoría Manejo de Conflictos

Respuestas

\section{DEFINICIÓN}

Las percepciones de las y los estudiantes sobre el manejo de conflictos fueron: desacuerdo de ideas. Un desacuerdo en una opinión o en una situación. Discrepancia en ciertos temas. No estar de acuerdo. No ponerse de acuerdo. Oposición entre dos o más personas. Llevarse la contraria.

Es un choque de ideas. Actitudes que chocan con los demás. Cuando chocan dos ideas. Lucha de ideas.

Conflicto en la universidad es cuando el estudiante hace lo que él quiere. No sentirse a gusto en un grupo en la universidad.

Es no dar solución a los problemas.

Es rivalidad.

Cuando no hay tolerancia.

Es un disgusto.

Una diferencia mal manejada.

\section{CARACTERÍSTICAS}

El conflicto en la universidad es cuando hay desacuerdos y diferencias en la realización de trabajos. En la universidad es donde se empieza a tener conflictos. Uno cede al cambio, o a las otras personas.

El conflicto al finalizar genera nuevas ideas. Lleva a la conversación y se generan nuevas ideas. Tiene consecuencias, positivas o negativas, dependiendo de las personas. Todos los días uno tiene conflictos, pero depende de cada uno verlo positivo o negativo.

Dentro del aula nunca hay conflicto sino roces. El conflicto en el aula se queda ahí, cuando sale, pasa. Es menor en el aula cuando uno escoge con quien trabajar.

El conflicto se da con uno mismo por decisiones que toma. No siempre es con otra persona, puede ser con uno mismo. La experiencia permite aprender y evita volver a tener conflictos con uno mismo.

El orgullo lleva a que el conflicto perdure. En el conflicto no hay valores bonitos.

El conflicto se crea por las discusiones de los padres. El perdón y el diálogo produce cambio en la familia y la fortalece.

El conflicto se ve en todas partes: laboral, académico, entre otros.

El conflicto fortalece las relaciones sociales. 
El conflicto permite conocer los errores.

Después de cada conflicto se debe arreglar las diferencias.

\section{CAUSAS}

La falta de respeto frente al pensar del otro. El irrespeto. No respetamos a las otras personas. No somos leales. El irrespeto. La deslealtad. El orgullo. La intolerancia es una de las causas del conflicto. Se da por no tolerar las ideas e imponer las propias. Imponer las ideas y no tolerar los comportamientos de los demás. La vanidad.

El conflicto se da por las diferencias de pensamiento. Diferencias en lo que se dice. Diversas formas de pensar. Diferentes ideologías. Formas no iguales de pensar. Distintas formas de intercambiar ideas. No aceptar los ideales. Diferencias de pensamiento. El conflicto se da por los diferentes pensamientos culturales.

No ponernos de acuerdo en un trabajo. No estar de acuerdo con lo que piensan los otros.

Tener desacuerdos con la pareja. No estar de acuerdo con otras personas.

El estrés. Asociar los problemas que tiene fuera con los de la universidad. Cuando uno tiene estrés.

Los conflictos en la universidad se dan por la diferencia entre las carreras.

La forma de hablar o de mirar genera conflicto.

No apoyar a otra persona. 1

La prepotencia de las personas.

1

Cuando una persona aporta más que otra.

Actuar mal a espaldas de la otra persona.

\section{Subcategoría. Conceptualización}

En el concepto de conflicto, los y las estudiantes otorgan prioridad al desacuerdo y choque de ideas con los demás y la influencia de las actitudes en las consecuencias que este pueda tener.

A partir de estos referentes, el sociólogo Vinyamata (2015) argumenta que el conflicto es un hecho personal, social, histórico y cultural e influye en la convivencia afectando las dimensiones psicosociales, definición que se adapta a las respuestas de los jóvenes. 
En síntesis, el conflicto es el producto de la conducta relacional humana y del resultado de convivir en sociedad con diversas ópticas sobre la manera de percibir la realidad, entendiendo que este no necesariamente implica o justifica cualquier forma de violencia.

Por otro lado, se recopila la diversidad de respuestas que en grado menor se revelan entendiendo el concepto de conflicto, como problemas en los que se presenta intolerancia, disgusto, diferencias mal manejadas, rivalidades y no dar soluciones a los mismos.

\section{Subcategoría. Características}

Se contextualiza el manejo de los conflictos fuera del aula de clase, especialmente en las diferencias y desacuerdos que se presentan en la realización de trabajos académicos que conducen a la conversación y a generar nuevas ideas. Así mismo se tiene una percepción dual del mismo, bien sea desde el punto de vista positivo o negativo, lo que depende de cómo cada uno lo aprecie y lo asuma. En este mismo orden de ideas, el conflicto posibilita el aprendizaje y evita reincidir en él; si el orgullo domina la relación interpersonal este persiste alterando la convivencia humana.

Después de dar a conocer los puntos de vista de las y los estudiantes con respecto a las características del manejo de conflictos, es pertinente asociarlas con Calderón (2009), quien hace mención a la Teoría de Conflictos de Galtung (1984:11), basándose en la triada: actitudes -se refieren a la percepción que se tiene del otro en una situación problema-; comportamiento -la manera de actuar de las personas-; y contradicción -reconocida por las diferencias de criterio y de interpretación real del conflicto-.

En menor nivel, otros estudiantes reúnen las concepciones del manejo del conflicto como algo que ocurre en todas partes, fortalece las diversas relaciones sociales, se presenta en el ambiente laboral y académico, permite conocer los errores, así como arreglar las diferencias luego de que se presenta.

\section{Subcategoría. Causas}

En alta proporción se identifican entre los y las estudiantes como formas de entender el manejo del conflicto, dos perspectivas. De una parte, el conflicto positivo, cuando existe la voluntad de una comunicación a través del diálogo directo, la escucha activa para interpretar y entender a la otra persona; se adhieren a estas opiniones esperando que se calmen los ánimos; quedarse callado y encontrar el momento indicado para resolver el problema manejando la asertividad. De otra parte, está el conflicto negativo, en el que, como su nombre mismo lo indica, no se aceptan las diferencias de pensamientos ideológicos y culturales, se imponen ideas y se asocian 
los problemas que se tienen fuera con los de la universidad. A las concepciones anteriores se añade como causa el irrespeto, la intolerancia, la deslealtad, el orgullo y el envenenarse uno mismo.

A partir de las opiniones sentidas en los grupos focales, se considera que Vinyamata (2015) se refiere a los conflictos como sucesos del diario vivir que forman parte del paisaje humano, se pueden tejer con los planteamientos de las y los estudiantes, quienes entienden el conflicto connatural con la vida misma. Los conflictos se relacionan con la satisfacción de las necesidades, se encuentran en relación con procesos de estrés y sensaciones de temor, y con el desarrollo de la acción que puede llevar o no hacia comportamientos agresivos y violentos.

A continuación, tomando los ítems de menor puntaje en las respuestas, se explican las voces de los y las estudiantes: los conflictos en la universidad fuera del aula de clase se dan por la diferencia entre las carreras, la forma de tratar a la otra persona, tanto en lo verbal o forma de hablar, como expresiones no verbales, en cuanto a la manera de mirar, la prepotencia de las personas. Creen que cuando una persona aporta más que otra, falla o actúa a espaldas y además le falta apoyo, la convivencia se hace difícil.

\section{Discusión}

Al ser las relaciones interpersonales el núcleo sobre el cual se centra la vida social y cultural, sabiamente y con razón Aristóteles afirmaba como característica del hombre ser un Zoon politicón, o sea, un animal social. Aunque esas interacciones del largo tejido humano han tenido y seguirán teniendo tropiezos, siguen siendo significativas y base para la existencia humana.

Dicho lo anterior, se inicia el proceso de discusión de resultados como uno de los aspectos importantes de la investigación, debido a las reflexiones que en ella se hacen sobre las categorías de análisis del proyecto: relación de amistad, relación de pareja y manejo de conflictos.

\section{Relaciones de amistad}

Se evidencia la forma natural y espontánea en que los y las estudiantes, en su mayoría, solventan sus posiciones y conceptos, manejando el respeto, la actitud de escucha y la comprensión en el momento del debate; aspectos congruentes con las respuestas que aparecen en la tabla 1. 
Predomina el enfoque humanístico psicosocial, por la disposición mental, la afectividad y expresión de emociones observadas a través de la comunicación verbal y no verbal utilizando por momentos un lenguaje coloquial; también por la forma de narrar ejemplificando con experiencias las vivencias fuera del aula en la institución académica, así como extrapolando a situaciones de amigos en otros contextos.

Sin embargo, pese a que la mayoría manifiesta una apertura para contribuir al buen desarrollo de la dinámica conversacional, algunos y algunas en mínima proporción reflejan falta de motivación, no dicen nada o evaden opiniones, lo que se puede inferir como timidez, introversión o poca confianza en la interacción, pese a haberse generado un clima cálido.

Finalmente se intuye, sin temor a equivocación, que el origen de su manera de pensar, sentir y asumir la mirada sobre la amistad es reflejo, de una parte, del proceso de socialización primaria (familia), en donde a través de modelos pedagógicos, los y las jóvenes interiorizan normas y reglas de comportamiento. De otra parte, por la socialización secundaria (entorno social y académico), donde se refuerzan los roles y estatus que van dando horizonte al proyecto de vida.

\section{Relaciones de pareja}

Se conjetura que cuando se habló de este aspecto interpersonal fue un momento apropiado para que el mayor porcentaje de estudiantes manifestara el sentir emocional a través del diálogo franco, sincero y comprensible, sobre la forma como entienden y quizá como experimentan las relaciones de pareja en su cotidianidad.

Las reacciones ante las preguntas guía de la discusión fueron homogéneas, dando lugar a sentimientos emotivos de halago y complacencia ante situaciones que les proporcionan entrega, cariño, afinidad, apoyo y amor. Debido al ambiente libre de prejuicios, por parte de los y las participantes, algunos jóvenes del género masculino se extrovertieron y expresaron sus tendencias sexuales y la forma como les afectan o no, añadiendo que hasta el momento no ha repercutido en la vida académica.

En menor porcentaje de estudiantes, surgió la apatía y hasta el desinterés para expresar y dejar fluir libremente las emociones. Aclarando que la muestra representativa osciló entre los 18 y 25 años, se vislumbra por parte de las investigadoras que la actitud observada puede deberse a su nivel de maduración, es decir, de los 18 a 21 años, el ajuste de identidad hace que se presente inestabilidad en el aspecto cognitivo (pensamiento) y afectivo (sentimiento) para dar respuestas sólidas; mientras que entre 21 y 25 años parecen tener una imagen clara de sí mismos y su relación con el otro, lo cual permite que sus respuestas sean más reflexivas. 
Por último, es necesario señalar que, en ínfima proporción, en situaciones de conflicto de pareja, se ven los y las jóvenes como protagonistas de las soluciones, lo que se cree representa una ganancia emocional y social, ya que al poner en práctica las habilidades relacionales del diálogo, la comprensión hacia el otro y la asertividad, se facilitará la positiva convivencia en un futuro.

\section{Manejo de conflictos}

De acuerdo con las respuestas de los y las estudiantes entrevistados, es grato encontrar el recurso lingüístico para definir empíricamente el conflicto, ya que la gran mayoría se aproxima a las definiciones sustentadas conceptualmente en la investigación. A su vez, la interacción comunicativa se mostró más fluida, manejando un lenguaje sencillo, claro, de apropiación. Quizá se deba a que en el medio social, institucional y en general del país se está dando el debate sobre el tema ampliamente.

La perspectiva frente a la visión del conflicto surge de una apreciación interna en los y las estudiantes, la cual es percibida por antivalores: irrespeto, deslealtad, intolerancia y rivalidad, entre otros. También es relevante destacar que utilizan como estrategias de negociación para la solución de los conflictos: el perdón, el diálogo y el acuerdo. Aspectos que pueden obedecer a circunstancias vividas o experiencias de sus propias realidades que de alguna manera les dejan huella, conduciéndolos a forjar una personalidad saludable y una posible convivencia armoniosa fuera del aula de clase.

\section{Conclusiones}

Se encuentra que en su mayoría los estudios y literatura sobre las relaciones de amistad, pareja y manejo de conflictos, en la educación primaria y media en Colombia, y algunos en el contexto internacional (España y México), están centrados en el aula de clase; y en escasa medida hay indagaciones fuera de ella. De esta forma se constituye el proyecto en un reto personal y académico para enriquecer el conocimiento y para compartirlo.

El estudio se realizó en la Facultad de Ciencias de la Salud y en la Facultad de Diseño, Comunicación y Bellas Artes. Por razones del calendario académico y disposición de tiempo, no se contempló hacer un estudio comparativo, pero se considera importante continuar en una segunda fase.

Las teorías sociológicas y psicológicas permitieron una correlación con las respuestas de las y los estudiantes en los grupos focales, y el procedimiento articulado y sistematizado de la investigación, contribuyendo al fortalecimiento del Proyecto Pedagógico Institucional y los diversos campos de formación. 
En relación a la disposición de las y los docentes para colaborar en el desarrollo de los talleres con los grupos focales, fue positiva; sin embargo, en el momento de solicitar el permiso de participación de las y los estudiantes surgieron algunos desfases en los tiempos.

Los testimonios de las y los estudiantes fueron valiosos en la medida en que permitieron, a través del diálogo abierto y espontáneo, la narración de vivencias donde afloraron emociones y sentimientos que, unidos a la discusión y reflexión en grupo, generaron interrogantes respecto a la manera de entender las relaciones.

El proyecto aporta luces a la comunidad académica respecto a las relaciones interpersonales externas al aula de clase, espacio que generalmente suele dejarse de lado por considerarse intrascendente, y muy probablemente por el afán de validar un compromiso centrado en el aula que, si bien es importante, invisibiliza el ambiente fuera de ella, donde fluyen ópticas cognitivas, afectivas y conductuales propias de la educación y la formación integral.

\section{Recomendaciones}

Es conveniente un mayor empoderamiento institucional del tema, especialmente con la comunidad académica disciplinar y socio-humanística de formación integral, a través de talleres y seminarios en habilidades comunicativas, que motiven y vinculen a docentes y estudiantes y les permitan resolver de forma propositiva las contingencias en el manejo de problemas tanto fuera del aula como en el interior de la misma.

Realizar proyectos con este mismo enfoque, teniendo en cuenta los cambios que a nivel de relaciones se han ido modificando por el uso de las Tics y las plataformas virtuales.

Diseñar propuestas de investigación comparativa a nivel interinstitucional.

\section{Referencias}

CALDERÓN C. P. (2009). Teoría de conflictos de Johan Galtung. Revista de Paz y Conflictos, (2), 10. Recuperado de http//www.redalyc.org/articulo.oa?id=205016389005

CALLEJA, M.W.H. (2011). «Hacia la optimización de las comunicacionesy su vinculación estratégica con las relaciones públicas como un proceso sistemático para la solución de los problemas empresariales». Perú: Universidad de Callao. Facultad de Ciencias Administrativas. Instituto de Investigaciones de la FCA. Recuperado de http//www.unac.edu.pe/images/documentos/organizacion/ vri/cdcitra/Informes_Finales_Investigacion/2011/Julio/IF_CALLEJA_MONTANI_FCA.PDF 
CAMPO C. Y LINARES, J.L. (2002). Sobrevivir a la pareja. Barcelona: Editorial Planeta.

CANO, B. (2008). La Ética arte de vivir 2. Talleres para formación de valores. Bogotá: Ediciones Paulinas.

GALTUN, J. (1984) ¡Hay alternativas! 4 caminos hacia la pazy la seguridad. Madrid: Tecnos.

GARRIDO G., A., REYES L. A. G., TORRES V. L. E. Y ORTEGA, S. P. (2008). Importancia de las expectativas de pareja en la dinámica familiar. Enseñanza e Investigación en Psicología, 13, (2), 232 Recuperado de http//www.redalyc.org/articulo.oa?id=29213203

GERRING, J. (2014). Metodología de las Ciencias Sociales. España: Alianza Editorial.

MORALES, M. M. (2005). «Análisis Psicosocial del Poder en las Relaciones de Género». Tesis doctoral. Departamento de Psicología Social y Metodología de las Ciencias del Comportamiento. España: Editorial Universidad de Granada

OBSERVATORIO PARA LA PAZ (2014). Elementos Conceptuales del Modelo Pacicultor. En «Pacicultura para una Educación Superior Inclusiva». Bogotá: Fundación Universitaria del Área Andina Observatorio para la Paz Ministerio de Educación Nacional.

ROGERS, C. (1980). El poder de la persona. México: Manual Moderno.

ROMERO IRIBAS, A.M. (2015). El Estatuto Antropológico de la Amistad y su Dimensión Social: perspectivas para el siglo XXI. Pamplona: Universidad de Navarra Facultad de Filosofía y letras Departamento de Filosofía. Recuperado de http//dadun.unav.edu/bitstream/10171/39719/1/ Tesis_AnaMariaRomeroIribas.pdf

SELLÈS, J. F. (2008). La educación de la amistad: una aproximación conceptual. Recuperado de http//site.ebrary.com.proxy.bidig.areandina.edu.co:2048/lib/bibliotecafuaasp/reader.action?docID $=10344991$

VINYAMATA, C. E. (2015). Revista de Paz y Conflictos. 8 (1). Instituto de la Paz y los Conflictos. España: Universidad de Granada. Recuperado de http//www.ugr.es/ revpaz/numeros/revpaz_8_1_completo.pdf

VÁSQUEZ, F. (2013). Destilar la información. (Un ejemplo seguido paso a paso). Bogotá: Universidad de la Salle Maestría en Docencia. 